Revue d'histoire de l'Amérique française

REYUE D.HISTOIRE DE L'AMÉRIQUE FRANÇAISE

\title{
LAPOINTE-ROY, Huguette, Charité bien ordonnée. Le premier réseau de lutte contre la pauvreté à Montréal au $19^{e}$ siècle. Montréal, Le Boréal, 1987. 330 p. 21,95 \$ \\ Sylvie Côté
}

Volume 41, numéro 1, été 1987

URI : https://id.erudit.org/iderudit/304529ar

DOI : https://doi.org/10.7202/304529ar

Aller au sommaire du numéro

Éditeur(s)

Institut d'histoire de l'Amérique française

ISSN

0035-2357 (imprimé)

1492-1383 (numérique)

Découvrir la revue

Citer ce compte rendu

Côté, S. (1987). Compte rendu de [LAPOINTE-ROY, Huguette, Charité bien ordonnée. Le premier réseau de lutte contre la pauvreté à Montréal au $19^{e}$ siècle. Montréal, Le Boréal, 1987. 330 p. 21,95 \$1. Revue d'histoire de l'Amérique française, 41(1), 85-87. https://doi.org/10.7202/304529ar d'utilisation que vous pouvez consulter en ligne.

https://apropos.erudit.org/fr/usagers/politique-dutilisation/ 
LAPOINTE-ROY, Huguette, Charité bien ordonnée. Le premier réseau de lutte contre la pauvreté à Montréal au 19e siècle. Montréal, Le Boréal, 1987. 330 p. $21,95 \$$

Bien que la pauvreté constitue un phénomène inhérent à toute société, l'historiographie s'y est peu intéressée jusqu'à maintenant. Le livre de Huguette Lapointe-Roy, Charité bien ordonnée. Le premier réseau de lutte contre la pauvreté à Montréal au 19e siècle, jette un premier regard sur l'assistance sociale au Québec. Publié par le Boréal, cet ouvrage décrit la mise sur pied, à Montréal, entre 1831 et 1871 , de diverses oeuvres d'assistance visant, selon l'auteure, à enrayer l'émigration. Huguette Lapointe-Roy nous livre ici une version remaniée de sa thèse de doctorat qu'elle a soutenue à l'Université Laval. L'ouvrage compte trois parties: les intervenants, les pauvres en institution et les services offerts aux pauvres à domicile; le lecteur trouvera aussi, en plus des notes et références, un index ainsi qu'une bibliographie. 
Deux écoles d'interprétation, l'une libérale et l'autre marxiste, dominent l'historiographie de l'assistance sociale. La première voit dans les changements sociaux et les nouvelles formes d'aide un signe de progrès; ainsi les religieuses et, plus tard, les travailleurs sociaux apparaissent comme des héros, soucieux du bien-être de toute la population. La seconde école, basée sur le concept de contrôle social, s'oppose à cette vision humanitariste du passé. A ses yeux, les mesures sociales prises par les autorités ne sont qu'un moyen pour dominer les classes inférieures et conserver l'ordre établi. Par conséquent, les orphelinats, les prisons et les hospices servent à la reproduction des valeurs bourgeoises et constituent un réseau d'institutions oppressif. LapointeRoy n'a pas choisi ce cadre d'analyse; elle se situe davantage dans la lignée du premier courant d'interprétation, réhabilitant ainsi l'intervention religieuse en matière de bien-être social. L'auteure démontre le rôle primordial joué par l'Église, particulièrement les congrégations religieuses féminines, pour venir en aide aux masses urbaines aux prises avec les problèmes sociaux liés à l'industrialisation. La première partie du titre, Charité bien ordonnée, exprime d'ailleurs très bien la position de l'auteure.

Dans la première partie, Lapointe-Roy traite des principaux intervenants montréalais dans la lutte contre la pauvreté. Par ordre d'importance, il s'agit du clergé (les Sulpiciens et Mgr Bourget), des congrégations religieuses féminines (les Soeurs Grises de Montréal et les Soeurs de la Providence), des laïcs et des bienfaiteurs qui agissent dans les associations telles que les Dames de la Charité et la Société Saint-Vincent-de-Paul et, en dernier lieu, des pouvoirs publics. Chaque groupe est étudié en fonction de la provenance de ses ressources financières, de sa contribution à l'établissement du réseau d'assistance et des rapports qu'il entretient avec les autres intervenants.

L'auteure aborde, dans la deuxième partie, la question des pauvres en institution qu'elle divise en deux catégories: les indigents inaptes au travail, soit les enfants abandonnés, les orphelins, les personnes âgées et les infirmes, puis les indigents aptes au travail, mais affectés par le chômage. Le chapitre 5 parle des conditions sociales et des conditions de vie des enfants abandonnés, orphelins et des vieillards dans différentes institutions: l'Hôpital général, l'Orphelinat Saint-Patrice, l'Hospice Saint-Joseph, l'Orphelinat catholique, l'Asile de la Providence, l'Orphelinat Saint-Alexis et l'Hospice Saint-Jérôme. On y apprend une foule de données intéressantes sur les habitudes alimentaires et hygiéniques de même que sur les règlements en vigueur dans ces institutions. Les services aux pauvres aptes au travail sont de trois Gidres: maison d'industrie, Confrérie du bien-public et services de placement. Lapointe-Roy catégorise les indigents en conformité avec l'idéologie libérale du $19 \mathrm{e}$ siècle qui valorise fortement le travail et l'effort individuel. Il aurait été souhaitable qu'elle se détache de cette vision du monde pour analyser ses informations.

La troisième partie de l'étude de Lapointe-Roy présente les différents services offerts aux pauvres à domicile. Suite à l'augmentation considérable de la clientèle des institutions d'assistance entre 1831 et 1846 , les intervenants ont vu la nécessité d'établir des services d'aide à domicile pour les démunis. C'est alors que sont apparus trois types de services: les visites à domicile, les dépôts des pauvres et l'oeuvre de la soupe. Les visites à domicile visaient à dépister les besoins et à fournir un appui moral et des conseils aux indigents, alors que les dépôts des pauvres devaient répondre à certains besoins matériels: 
aliments, vêtements, articles ménagers et bois de chauffage. Quant à l'oeuvre de la soupe, elle répondait surtout aux besoins des hommes incapables de cuisiner.

Lapointe-Roy nous fournit des renseignements très précieux sur l'assistance sociale au 19e siècle, mais uniquement sur le mode descriptif, laissant de côté les aspects analytiques et critiques. Ainsi lorsqu'elle parle de l'Orphelinat Saint-Alexis, qui a été étudié par Bettina Bradbury, elle ne fait aucunement mention des données de cette dernière ni de ses hypothèses de travail. Bradbury précise que l'orphelinat accueillait beaucoup d'enfants dont les parents étaient vivants, ce qui lui permet de conclure que le recours à l'orphelinat constituait une stratégie familiale pour surmonter certains problèmes comme le chômage, la maladie ou la mort. Une fois la crise terminée, les enfants retournaient chez leurs parents. L'orphelinat apparaît alors comme une institution qui vient en aide à la famille et qui en assure la survie contrairement à ce qu'affirme Lapointe-Roy, qui la dépeint comme une oeuvre recueillant les orphelins de père et de mère et les enfants abandonnés par de mauvais parents. De plus, l'ouvrage ne comprend, en guise de conclusion, qu'un court texte d'une page, ce qui est nettement insuffisant pour un ouvrage de cette envergure.

Charité bien ordonnée est basé sur une documentation abondante: l'auteure a consulté une multitude de fonds d'archives au Québec et en France, dont ceux des communautés religieuses. Les archives de la ville de Montréal de même que les journaux de l'époque et les publications gouvernementales ont aussi été dépouillés.

L'écriture de Lapointe-Roy est fort agréable et elle plaira sûrement à un large public puisqu'on lui a retranché les passages à caractère méthodologique. Fidèle à sa tradition, le Boréal nous offre un ouvrage d'une grande qualité matérielle. Sur la couverture apparaît une toile de Napoléon Bourassa, intitulée La pauvreté, qui témoigne bien des conditions de vie des indigents d'autrefois. De plus, une série de photographies intercalées dans le texte illustrent les propos de l'auteure. Ce livre ouvre la voie à d'autres recherches dans le domaine de l'assistance sociale et met à jour les efforts déployés par les différents acteurs sociaux pour aider les pauvres gens lors de l'industrialisation et de l'urbanisation de la ville de Montréal au siècle dernier. 